\title{
Fabrication of Integrated Device Comprising Flexible Dye-sensitized Solar Cell and Graphene-doped Supercapacitor
}

\author{
Wei-Ching Chuang, ${ }^{1}$ Chun-Ying Lee, ${ }^{1}$ Tung-Lung Wu, ${ }^{2 *}$ Chi-Ting Ho, ${ }^{3}$ \\ Yu-Yao Kang, ${ }^{4}$ Chun-Hsien Lee, ${ }^{1}$ and Teen-Hang Meen ${ }^{4 * *}$ \\ ${ }^{1}$ Institute of Electro-Optical and Materials Science, National Formosa University, Huwei 632, Yunlin, Taiwan \\ ${ }^{2}$ School of Mechanical and Automotive Engineering, Zhaoqing University, Zhaoqing 516260, Guangdong, China \\ ${ }^{3}$ Department of Mechanical Design Engineering, National Formosa University, Huwei 632, Yunlin, Taiwan \\ ${ }^{4}$ Department of Electronic Engineering, National Formosa University, Huwei 632, Yunlin, Taiwan
}

(Received January 16, 2020; accepted May 1, 2020)

Keywords: supercapacitor, activated carbon, dye-sensitized solar cell

Currently, flexible surfaces are becoming increasingly common in electronic devices. Electronic devices fabricated on flexible substrates are considered to have potential. Flexible supercapacitors have a long cycle life, high power density, stability, and flexibility. In this study, a flexible supercapacitor was prepared using activated carbon doped with graphene and was integrated with a flexible dye-sensitized solar cell (DSSC) to fabricate an energy conversion/storage device. A baking method was used to make the activated carbon doped with graphene powder, and a paste of this powder was deposited for use as an electrode of the supercapacitor by spin coating. A press machine was used to transfer a sintered $\mathrm{TiO}_{2}$ film from a quartz substrate to an indium tin oxide/polyethylene naphthalate (ITO/PEN) plastic substrate to fabricate a flexible DSSC. The supercapacitor doped with $0.05 \mathrm{wt} \%$ graphene had the highest capacitance, which was $218 \mathrm{~F} / \mathrm{g}$ with a charge-discharge efficiency of $85.29 \%$. The flexible supercapacitor maintained its capacitance well even when it was bent. A flexible DSCC integrated with a supercapacitor was fabricated to successfully power an LED.

\section{Introduction}

Environmental issues have increased the importance of researching efficient and sustainable energy conversion and storage equipment. At present, convenient portable devices for energy harvesting and storage devices are being studied. ${ }^{(1,2)}$ Supercapacitors are very suitable for use as energy storage components because of their long cycle life, high energy conversion efficiency, low equivalent series resistance, and fast charging/discharging. ${ }^{(3,4)}$ An electric double-layer capacitor (EDLC) is a type of supercapacitor. In an EDLC, the charge is stored at the surface of the electrode by the reversible absorption/desorption of ions to form an electrical double layer. ${ }^{(5)}$ An EDLC can be considered as an alternative to rechargeable batteries in applications that require fast energy harvesting or high-power delivery. However, the restriction of charge storage to the surface of supercapacitors makes their energy density much lower than 
that of batteries. Therefore, many researchers are trying to increase their energy density. ${ }^{(6-9)}$ The main challenge of supercapacitors is to develop them with high energy densities while maintaining their characteristics of long cycle life, fast charging/discharging, and high power. ${ }^{(10-12)}$ In addition, dye-sensitized solar cells (DSSCs) have attracted much attention since they have a simple manufacturing process, use low-cost materials, and have a light weight, flexibility, good photocurrent conversion efficiency, and a short energy payback time. DSSCs offer the possibility of designing flexible solar cells with color, transparency, and different shapes, providing an efficient and easily implemented technology for future energy supply. ${ }^{(13-16)}$ In this study, a flexible supercapacitor was prepared using activated carbon doped with graphene and was integrated with a flexible DSSC to fabricate an energy conversion/storage device. The materials and process of the integrated device were investigated in this study. Finally, the integrated flexible device was connected to a circuit with an LED and successfully powered the LED. A brief discussion of the application of the proposed device to sensing is also given.

\section{Experimental}

\subsection{Preparation of $\mathrm{TiO}_{2}$ photoanodes for DSCCs by doctor-blade and pressure transfer methods}

$\mathrm{TiO}_{2}$ powder, tert-butyl, and deionized water were mixed to form a paste. The paste was coated on an indium tin oxide/polyethylene naphthalate (ITO/PEN) plastic substrate by the doctor-blade method. To form a pressure-transferred thin film, the paste was coated on a quartz substrate by the doctor-blade method as an adhesion layer, then sintered at $450{ }^{\circ} \mathrm{C}$ for $30 \mathrm{~min}$ to form a transfer layer. Subsequently, the $\mathrm{TiO}_{2}$ thin film on the quartz substrate was placed in face-to-face contact with the ITO/PEN substrate, and pressure-transferred from the quartz substrate to the ITO/PEN substrate. The $\mathrm{TiO}_{2}$ thin film was used as a photoanode.

\subsection{Synthesis of graphene-doped carbon paste for supercapacitor}

$2 \mathrm{wt} \%$ polyvinyl butyral, $9 \mathrm{~g}$ of dimethylacetamide, different proportions of graphene, and $2 \mathrm{~g}$ of activated carbon were mixed and stirred for $3 \mathrm{~h}$. This graphene-doped toner was annealed at $300{ }^{\circ} \mathrm{C}$ for $2 \mathrm{~h}$. Then $1 \mathrm{~g}$ of the graphene-doped toner was stirred with $30 \mathrm{wt} \%$ carbon black, $9 \mathrm{~g}$ of dimethylacetamide, and $2 \mathrm{wt} \%$ polyvinyl butyral for $3 \mathrm{~h}$ to synthesize the graphene-doped carbon paste.

\subsection{Preparation of carbon electrode for supercapacitor}

The cleaned ITO/PEN substrate was coated with different weight percentages of graphene-doped carbon paste as a cathode using a spin coater at $1000 \mathrm{rpm}$ for $10 \mathrm{~s}$, then at 2000 rpm for $20 \mathrm{~s}$. The graphene-doped carbon paste was coated on foamed nickel as an anode. The ITO/PEN and the foamed nickel were dried in an oven to remove the organic solvent, thereby completing the preparation of the carbon electrode of the supercapacitor. 


\subsection{Fabrication of integrated device with flexible supercapacitor and DSSC}

\subsubsection{Fabrication of anode and cathode}

First, the ITO/PEN substrate was shaken in an ultrasonic cleaner using methanol and deionized water for $10 \mathrm{~min}$ and placed in an oven to dry. Second, the paste of titanium dioxide was mixed with titanium dioxide P25, deionized water, and butanol at a concentration ratio of $10 \mathrm{wt} \%$, and the mixture was stirred using a magnetic stirrer to form a titanium dioxide slurry. Third, a titanium dioxide anode was fabricated as follows. The titanium dioxide slurry was applied on the quartz substrate by the doctor-blade method, then the substrate was heated to $450{ }^{\circ} \mathrm{C}$ for $30 \mathrm{~min}$. After that, the titanium dioxide film was transferred to the ITO/PEN substrate by the pressure transfer method, where the film area was $0.25 \mathrm{~cm}^{2}(0.5 \mathrm{~cm} \times 0.5 \mathrm{~cm})$. Lastly, the cathode was fabricated by using a gold-plating machine to plate a $1 \mathrm{~nm}$ platinum (PT) layer on the ITO/PEN substrate.

\subsubsection{Mixing the dye and electrolyte}

An N3-ruthenium complex dye with a concentration of $0.3 \mathrm{mM}$ and butanol were mixed

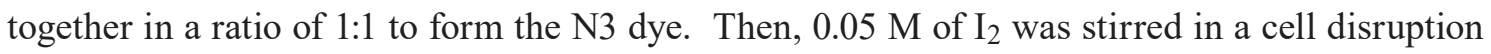
machine with $0.1 \mathrm{M}$ of LiI, $1 \mathrm{M}$ of 1,2-dimethyl-3-propylimidazolium iodide (DMPII), and $0.5 \mathrm{M}$ of 4-tert-butylpyridin (TBP) in 3-methoxypropionitrile (MPN) to form the electrolyte.

\subsubsection{DSSC encapsulation}

The anode was immersed in the dye for $2 \mathrm{~h}$, then taken out. Holes were drilled in the cathode to inject the electrolyte. Then, a thermoplastic film was used to heat and bond the soaked anode and cathode at about $100{ }^{\circ} \mathrm{C}$ on a heating platform. Electrolyte was injected into the holes of the cathode, and the holes were sealed with optical glue to complete the DSCC.

\subsubsection{Supercapacitor encapsulation}

First, a hole was drilled in the upper left corner of the cathode substrate (the anode was a carbon electrode coated with nickel foam), then a conductive separator was placed midway between the two poles. The main function of the conductive separator is to isolate the two poles to prevent the self-discharge and short circuit of the supercapacitor. The two poles were encapsulated with optical glue, then $6 \mathrm{M} \mathrm{KOH}$ electrolyte was injected into the drilled hole, and finally optical glue was used to glue the hole to complete the encapsulation.

\subsection{Integration of DSSC and supercapacitor}

The integration of the DSSC and supercapacitor involved directly merging the two cathodes. First, the ITO/PEN substrate was cut to $4.5 \mathrm{~cm} \times 3 \mathrm{~cm}$, and the right side $(3 \mathrm{~cm} \times 1.5 \mathrm{~cm})$ was coated with $1 \mathrm{~nm}$ PT, which served as the cathode of the DSSC. The electrode was then 
spin-coated with a supercapacitor graphene-doped carbon cathode electrode. Refer to Sect. 2.3 for the coating method. Note that a working area of $3 \mathrm{~cm} \times 3 \mathrm{~cm}$ remained on the left of the cut ITO/PEN substrate. The production and encapsulation of the DSSC and supercapacitor anode were then completed by the previous steps described in Sects. 2.1-2.3.

\subsection{Characterization of flexible supercapacitor, DSCC, and integrated device}

Cyclic voltammetry (CV) was performed to detect the type of current by changing the applied voltage. Each analyte had a different redox potential, and its specific capacitance was calculated using

$$
C=\frac{d Q}{d V}=\frac{1}{m v \Delta V} \int_{V_{1}}^{V_{2}} I(V) d V
$$

where $\int_{V_{1}}^{V_{2}} I(V) d V$ is the area of hysteresis, $v$ the scanning speed, $\Delta V$ the difference in voltage, and $m$ the total weight of the electrode material. The CV curve of supercapacitance is rectangular. The specific curve was changed by the measurement system in accordance with the type of applied voltage and the rectangular curve. In this study, the supercapacitance of the electrolyte was examined by a charge-discharge test with a constant current and the efficiency of the method was calculated using

$$
C=\frac{Q_{d e h}}{Q_{c h}} \times 100 \%=\frac{t_{d c h}}{t_{t c h}} \times 100 \%
$$

Here, $Q_{d e h}$ is the discharge capacity, $Q_{c h}$ the charge capacity, $t_{d c h}$ the time of discharge, and $t_{c h}$ the time of charging. ${ }^{(12,13)}$ Structural analysis was carried out using X-ray diffraction (XRD). The surface morphology of the samples was observed by field-emission scanning electron microscopy (FE-SEM). Ultraviolet and visible light (UV-Vis) absorption spectra of the samples were observed using a UV-Vis spectrophotometer. The current-voltage characteristics of the samples were measured using a Keithley 2400 source meter and were determined under simulated sunlight with white light PL of intensity $100 \mathrm{~mW} / \mathrm{cm}^{2}$. In the incident photon conversion efficiency (IPCE) measurement, a xenon lamp (Oriel 66150, $75 \mathrm{~W}$ ) was used as the light source. A chopper and a lock-in amplifier were used for phase-sensitive detection. The IPCE data were collected by shining a monochromatic light on the solar cells with wavelengths from 300 to $800 \mathrm{~nm}$ using a Keithley 2400 source meter. In the characterization of the integrated device, the solar simulator simulates sunlight, which is irradiated to the DSSC. The DSSC converts the energy to power that is supplied to the LED. The excess energy is transferred to the supercapacitor for storage. When there is no sunlight, the supercapacitor is used to supply LED power. Figure 1 shows the circuit diagram of the integrated device with an LED and a resistance. It uses four DSSCs and two integrated devices to store energy. To control the current flowing into the LED, a current-limiting resistor was added before the LED, then the power supply time of the capacitor to the LED was measured. 


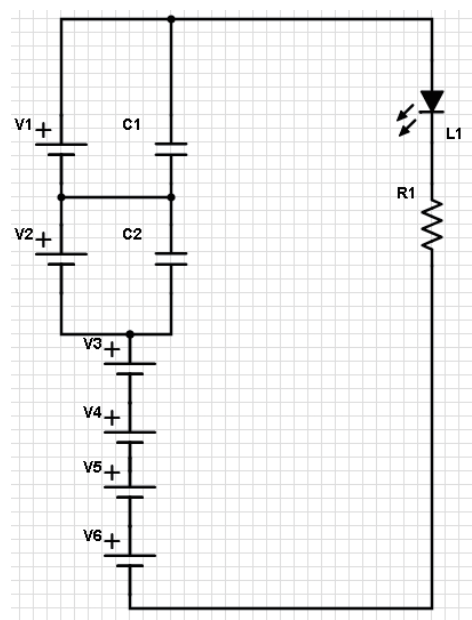

Fig. 1. Circuit diagram of integrated device with an LED and current-limiting resistor.

\section{Results and Discussion}

In this study, two different processing methods were used to make photoanodes of the DSCC. One was a $\mathrm{TiO}_{2}$ thin film directly scraped by the doctor-blade method. The other was prepared by the pressure transfer method. Figure 2 shows top- and side-view FE-SEM images of $\mathrm{TiO}_{2}$ thin films fabricated with the doctor-blade and pressure transfer methods. After the pressure treatment, the surface of the $\mathrm{TiO}_{2}$ thin film was flat. The thicknesses of the $\mathrm{TiO}_{2}$ thin films were about 11 and $9 \mu \mathrm{m}$ for the doctor-blade and pressure transfer methods, respectively.

Figure 3 shows UV-Vis absorption spectra of $\mathrm{TiO}_{2}$ thin films prepared by the doctor-blade and pressure transfer methods after the adsorption of $\mathrm{N} 3$ dye. The absorbance of the $\mathrm{TiO}_{2}$ thin film prepared by pressure transfer is higher than that of the film prepared by the doctorblade method. Figure 4 shows the current-voltage characteristics of DSSCs prepared by the doctor-blade and pressure transfer methods. The short-circuit current density $\left(J_{S C}\right)$, the open circuit voltage $\left(V_{O C}\right)$, the fill factor $(F F)$, and the overall conversion efficiency $(\eta)$ are listed in Table 1. From the results in Fig. 4 and Table 1, the conversion efficiency of the DSSC prepared by pressure transfer is higher than that of the DSCC prepared by the doctor-blade method. This may be due to the greater crystallization and higher quality of the former film under pressure and heat treatment.

Figure 5 shows the IPCE characteristic curves of DSSCs prepared by the two methods. The results of IPCE analysis indicate the number of incident photons inside the solar cells and their contribution to efficiency. All the IPCE spectra are similar in shape, and the IPCE of the DSSC prepared by the pressure transfer method is higher than that of the DSSC prepared by the doctor-blade method, especially from 400 to $700 \mathrm{~nm}$. This is consistent with the UV-Vis absorption spectra and current-voltage characteristics. It also proves that the use of higher quality $\mathrm{TiO}_{2}$ thin films as photoanodes can improve dye adsorption and increase the number of incident photons inside the solar cells. 

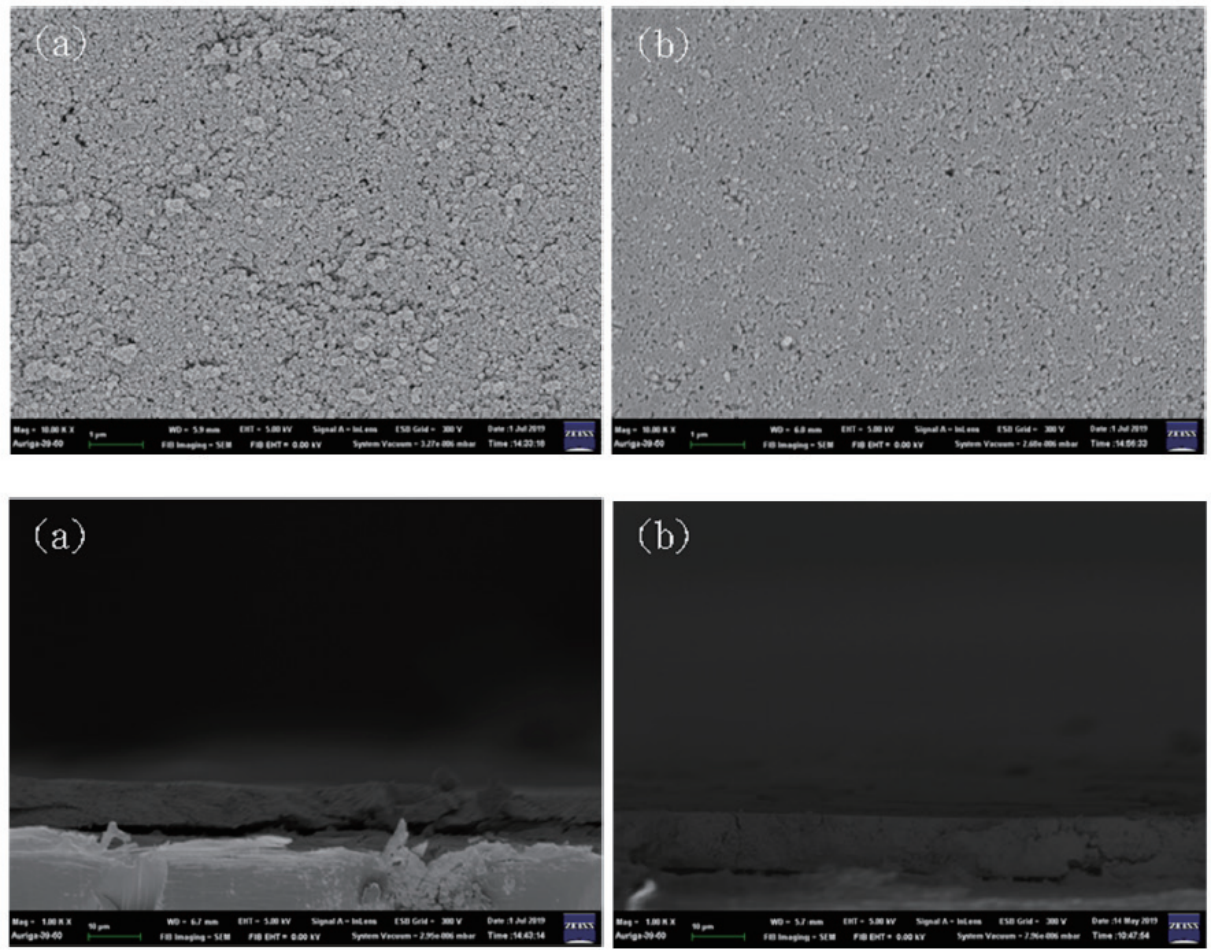

Fig. 2. Top- and side-view FE-SEM images of $\mathrm{TiO}_{2}$ thin films prepared by (a) doctor-blade method and (b) pressure transfer method.

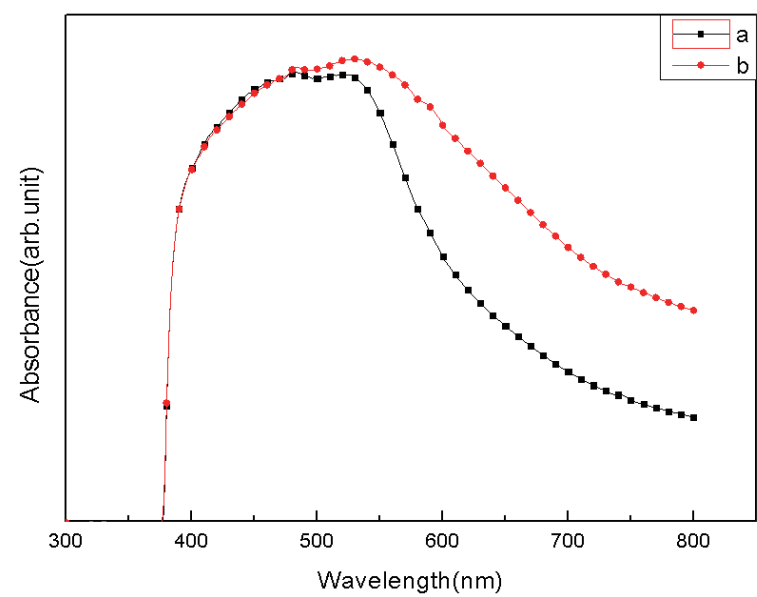

Fig. 3. (Color online) UV-Vis absorption spectra of $\mathrm{TiO}_{2}$ thin films prepared by (a) doctor-blade method and (b) pressure transfer method after N3 dye adsorption.

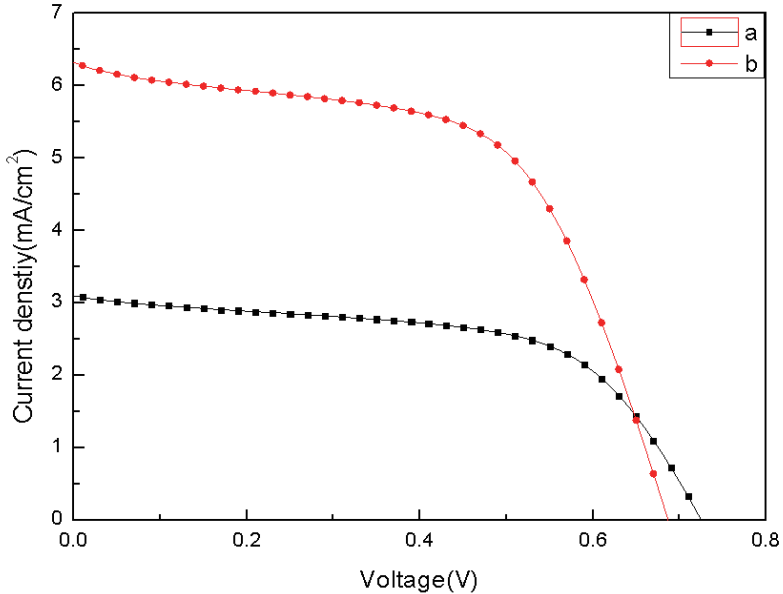

Fig. 4. (Color online) Current-voltage curves of DSSCs prepared by (a) doctor-blade method and (b) pressure transfer method.

Table 1

Current-voltage characteristics of DSSCs: (a) doctor-blade method and (b) pressure transfer method.

\begin{tabular}{lcccccc}
\hline Sample & $V_{O C}(\mathrm{~V})$ & $J_{S C}\left(\mathrm{~mA} / \mathrm{cm}^{2}\right)$ & $V_{m}(\mathrm{~V})$ & $J_{m}\left(\mathrm{~mA} / \mathrm{cm}^{2}\right)$ & $F F(\%)$ & $\eta(\%)$ \\
\hline $\mathrm{a}$ & 0.73 & 3.10 & 0.55 & 2.40 & 58.74 & 1.32 \\
$\mathrm{~b}$ & 0.69 & 6.32 & 0.50 & 5.07 & 58.37 & 2.54 \\
\hline
\end{tabular}




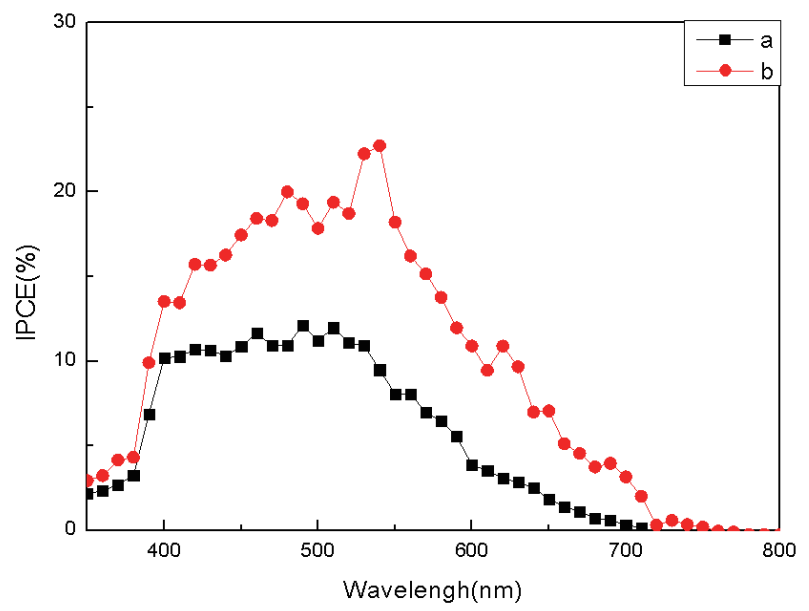

Fig. 5. (Color online) IPCE characteristic curves of DSSCs prepared by (a) doctor-blade method and (b) pressure transfer method.

Figure 6 shows FE-SEM images of electrode surfaces for supercapacitors with different weight percentages of graphene. The electrode with $0.05 \mathrm{wt} \%$ graphene had the most uniform grains. Adding graphene in electrodes can bring about excellent electrical conductivity owing to its high electron mobility and low resistivity. The specific capacitance value and charge-discharge efficiency were improved by changing the amount of graphene. When $0.07 \mathrm{wt} \%$ graphene was added in the electrode, the concentration was too high and the surface of the carbon electrode cracked. Figure 7 shows a CV analysis chart of supercapacitors with different weight percentages of graphene. The area of hysteresis increased when the amount of graphene increased. When the weight percentage of graphene reached $0.05 \%$, the greatest hysteresis was found. Figure 8 shows the charge-discharge graph of supercapacitors with different weight percentages of graphene. The CV and charge-discharge parameters of the supercapacitors obtained from Figs. 7 and 8 with different weight percentages of graphene are also listed in Table 2. The optimal amount of graphene added to the carbon electrode is $0.05 \mathrm{wt} \%$, for which the specific capacitance is $218 \mathrm{~F} / \mathrm{g}$ and the charge-discharge efficiency $\eta$ is $85.29 \%$. These results are consistent with the FE-SEM images.

Figure 9 shows a capacitance retention analysis chart of supercapacitors with different weight percentages of graphene. The graphene supercapacitors maintain good capacitance values after $1000 \mathrm{CV}$ cycles. The optimal amount of graphene added in the carbon electrode was also $0.05 \mathrm{wt} \%$. To apply this supercapacitor on a flexible device, a bending test was carried out to examine the changes in capacitance and charge-discharge efficiency. Figure 10 shows the bending test of the supercapacitor. Figure 11 is the $\mathrm{CV}$ analysis chart of the supercapacitor with $0.05 \mathrm{wt} \%$ graphene under bending. Figure 12 is the charge-discharge graph of the supercapacitor with $0.05 \mathrm{wt} \%$ graphene under bending. It is found that the supercapacitor retains its high quality under bending. Since applications of advanced materials for electronic and optical sensors have been fast-developing fields in recent years, the flexibility of these sensors gives them the potential to be deployable. Therefore, this supercapacitor can be applied as a flexible device for energy storage. 

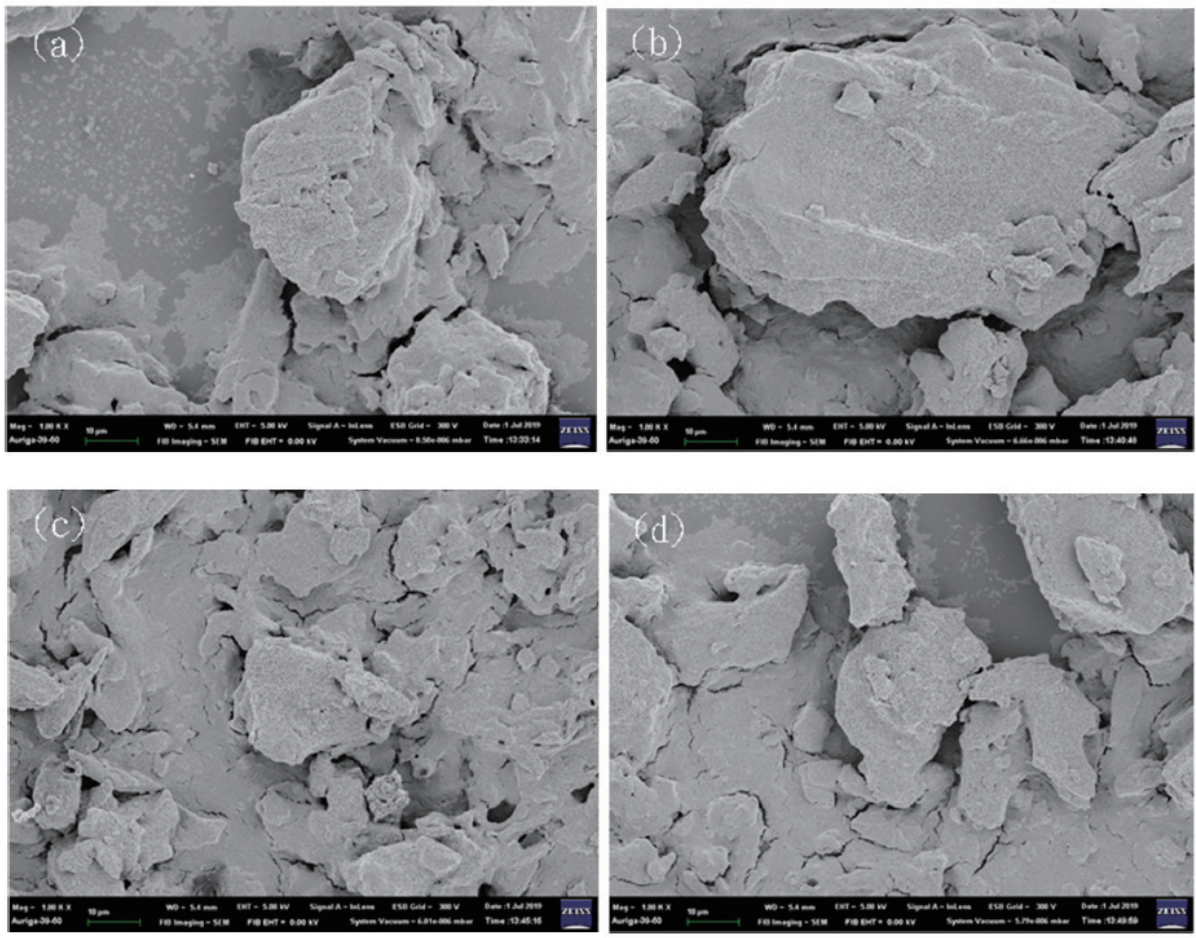

Fig. 6. FE-SEM images of electrode surfaces for supercapacitors with different weight percentages of graphene: (a) $0.01 \%$, (b) $0.03 \%$, (c) $0.05 \%$, (d) $0.07 \%$.

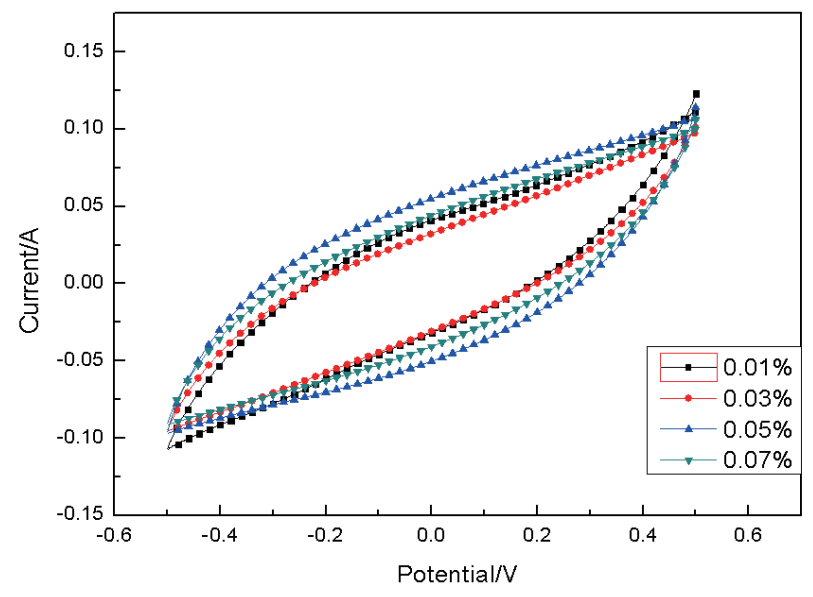

Fig. 7. (Color online) CV analysis chart of supercapacitors with different weight percentages of graphene: (a) $0.01 \%$, (b) $0.03 \%$, (c) $0.05 \%$, (d) $0.07 \%$.

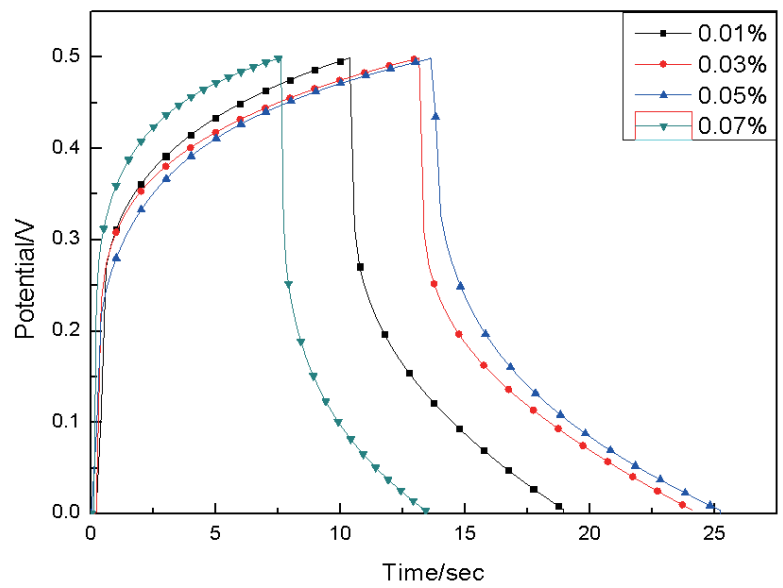

Fig. 8. (Color online) Charge-discharge graph of supercapacitors with different weight percentages of graphene: (a) $0.01 \%$, (b) $0.03 \%$, (c) $0.05 \%$, (d) $0.07 \%$.

Table 2

$\mathrm{CV}$ and charge-discharge parameters of supercapacitors obtained from Figs. 7 and 8 with different weight percentages of graphene: (a) $0.01 \%$, (b) $0.03 \%$, (c) $0.05 \%$, (d) $0.07 \% \mathrm{wt} \%$.

\begin{tabular}{lccc}
\hline $\begin{array}{l}\text { Graphene } \\
(\mathrm{wt} \%)\end{array}$ & $\begin{array}{c}\text { Charge-discharge } \\
\text { efficiency, } \eta(\%)\end{array}$ & $\begin{array}{c}\text { Specific capacitance } \\
(\mathrm{F} / \mathrm{g})\end{array}$ & $\int_{V_{1}}^{V_{2}} I(V) d V$ \\
\hline 0.01 & 82.62 & 189.66 & $9.15 \times 10^{-2}$ \\
0.03 & 83.36 & 200.08 & $1.04 \times 10^{-1}$ \\
0.05 & 85.29 & 218 & $1.12 \times 10^{-1}$ \\
0.07 & 76.21 & 206.48 & $1.10 \times 10^{-1}$ \\
\hline
\end{tabular}




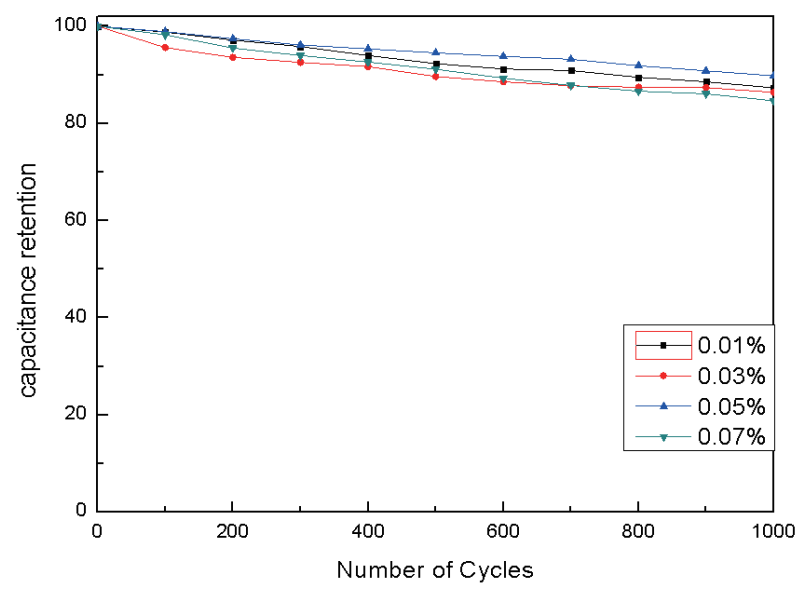

Fig. 9. (Color online) Capacitance retention analysis chart of supercapacitors with different weight percentages of graphene: (a) $0.01 \%$, (b) $0.03 \%$, (c) $0.05 \%$, (d) $0.07 \%$.

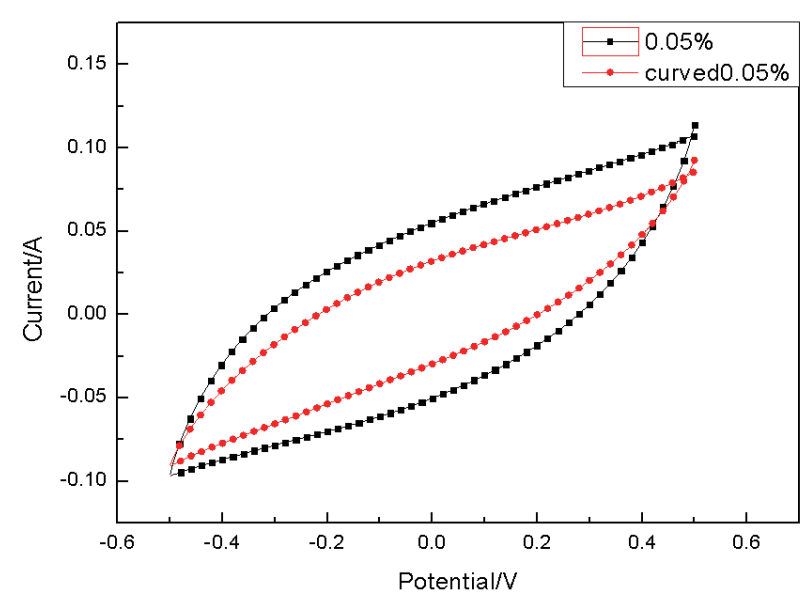

Fig. 11. (Color online) CV analysis chart of supercapacitor with 0.05 wt $\%$ graphene under bending test.

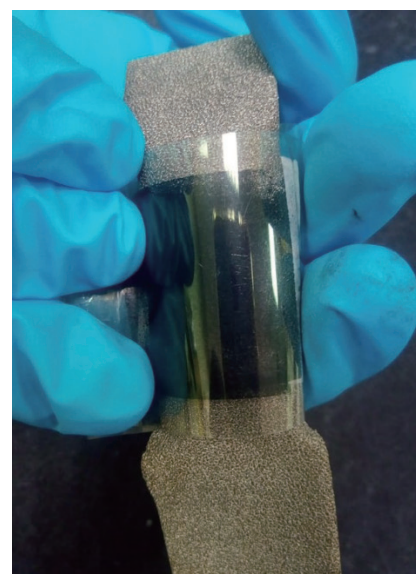

Fig. 10. (Color online) Bending test of supercapacitor.

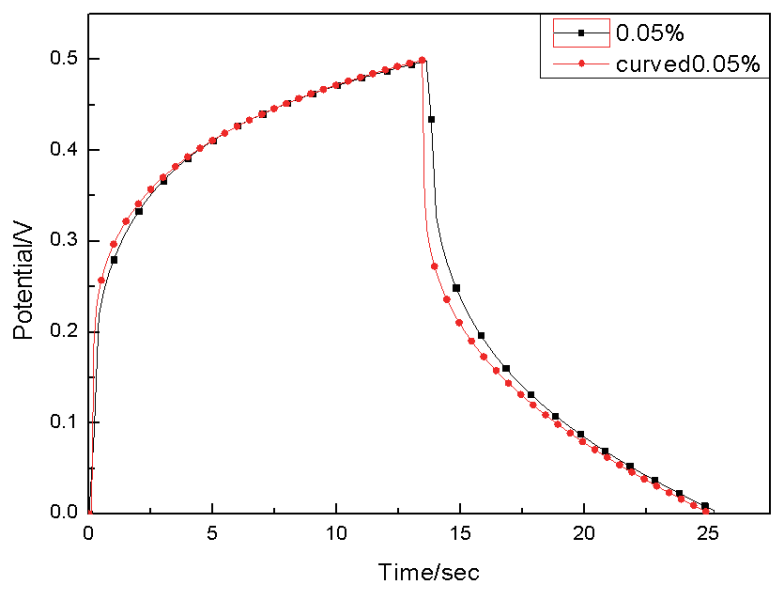

Fig. 12. (Color online) Charge-discharge graph of supercapacitor with $0.05 \mathrm{wt} \%$ graphene under bending test.

Figure 13 shows diagrams of this integrated device in operation. The sunlight simulator simulates sunlight, which is irradiated on the DSCC, and solar energy is generated by the DSCC. The excess energy is transmitted to the supercapacitor for storage. When there is no sunlight, the supercapacitor is used as a power supply to drive the LED. This circuit uses multiple dye-sensitized batteries to generate solar energy. To control the current flowing into the LED, a current-limiting resistor is added before the LED. The figure shows the process of charging and discharging the integrated device. There is no sunlight in the initial state and the capacitor voltage is $0 \mathrm{~V}$, as shown in Fig. 13(a). The solar light irradiates the DSCC to generate energy to charge the supercapacitor, as shown in Fig. 13(b). After charging the supercapacitor 

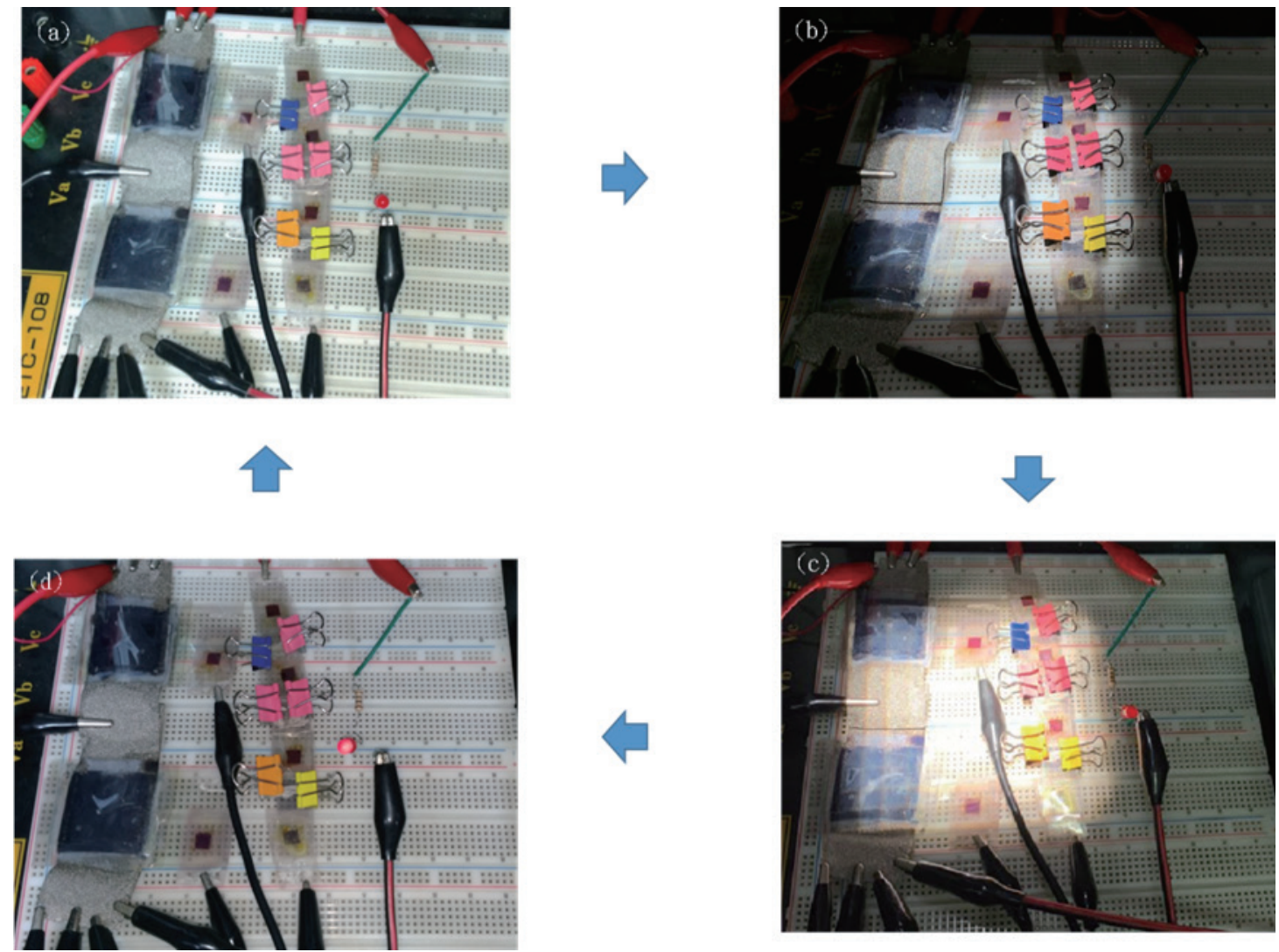

Fig. 13. (Color online) Diagrams of integrated device in operation.

for a few seconds, the capacitor voltage is higher than the LED operating voltage, and the LED is illuminated, as shown in Fig. 13(c). At the same time, the sunlight simulator is removed. After a period of time, the stored energy of the supercapacitor gradually decreases, as shown in Fig. 13(d). When the voltage is lower than the operating voltage of the LED, the LED is extinguished, and the process returns to Fig. 13(a). The integrated device comprising the flexible DSSC and graphene-doped supercapacitor was fabricated successfully in this study. The innovation of this study is the integration of a supercapacitor and a DSCC into an independent power source. DSCCs can be used for power generation, while the supercapacitor stores the electric energy. Internet of Things (IoT) sensors usually require little power because they may monitor data once every few minutes or hours. This integrated device is very suitable for powering such applications and can also maintain the stability of IoT sensors. When the sensor is working, it is powered by this integrated device. When the sensor is not working, the supercapacitor is recharged for the next startup.

\section{Conclusions}

In this study, a flexible supercapacitor was prepared using activated carbon doped with graphene, and was integrated with a flexible DSSC to fabricate an energy conversion/storage 
device. The supercapacitor doped with $0.05 \mathrm{wt} \%$ graphene had the highest capacitance and charge-discharge efficiency, with a capacitance of $218 \mathrm{~F} / \mathrm{g}$ and a charge-discharge efficiency of $85.29 \%$. The flexible supercapacitor maintained its capacitance well even when bent. A flexible DSCC integrated with a supercapacitor was fabricated and successfully powered an LED.

\section{References}

1 C. H. Ng, H. N. Lim, S. Hayase, I. Harrison, A. Pandikumar, and N. M. Huang: J. Power Sources 296 (2015) 169. https://doi.org/10.1016/j.jpowsour.2015.07.006

2 C. T. Chien, P. Hiralal, D. Y. Wang, I. S. Huang, C. C. Chen, C. W. Chen, and G. A. J. Amaratunga: Small 11 (2015) 2929. https://doi.org/10.1002/smll.201403383

3 J. S. Bonso, A. Rahy, S. D. Perera, N. Nour, O. Seitz, Y. J. Chabal, K. J. Balkus Jr., J. P. Ferraris, and D. J. Yang: J. Power Sources 203 (2012) 227. https://doi.org/10.1016/j.jpowsour.2011.09.084

4 L. Yuan, X. Xiao, T. Ding, J. Zhong, X. Zhang, Y. Shen, B. Hu, Y. Huang, J. Zhou, and Z. L. Wang: Angrew. Chem. Int. Ed. 51 (2012) 4934. https://doi.org/10.1002/anie.201109142

5 C. Zhao, Q. Wang, H. Zhang, S. Passerini, and X. Qian: ACS Appl. Mater. Interfaces 8 (2016) 15661. https:// doi.org/10.1021/acsami.6b04767

6 T. Cetinkaya and R. A. W. Dryfe: J. Energy Power Sources 408 (2018) 91. https://doi.org/10.1016/ j.jpowsour.2018.10.072

7 T. X. Tran, H. Choi, C. H. Che, J. H. Su, I. G. Kim, S. M. Lee, J. H. Kim, and J. B. In: ACS Appl. Mater. Interfaces 10 (2018) 39777. https://doi.org/10.1021/acsami.8b14678

8 M. O. Yanik, E. A. Yigit, Y. E. Akansu, and E. Sahmetlioglu: Energy 138 (2017) 883. https://doi.org/10.1016/ j.energy.2017.07.022

9 K. Urita, C. Urita, K. Fujita, K. Horio, M. Yoshida, and I. Moriguchi: Nanoscale 40 (2017) 15643. https://doi. org/10.1039/C7NR05307J

10 C. Yu, G. Meisong, Z. Miaomiao, Y. Yanan, and H. Jingbo: J. Nanosci. Nanotechnol. 20 (2020) 2402. https:// doi.org/10.1166/jnn.2020.17377

11 L. Shubra, J. Akanksha, S. Gurmeet, and S.R. Kishore: Electrochimica Acta 328 (2019) 130576. https://doi. org/10.1016/j.electacta.2019.135076

12 L. Jing, W. Ning, T. Jiarui, Q. Weizhong, and C. Wei: Adv. Funct. Mater. 28 (2018) 187360 . https://doi. org/10.1002/adfm.201806153

13 M. I. Chandra, S. Shalini, S. Sudipta, S. Pankaj, and B. Lal: Sol. Energy 194 (2019) 952. https://doi.org/10.1016/ j.solener.2019.10.090

14 V. Shanmuganathan, L. W. Hsun, T. Hsisheng, and L. Y. Lang: ACS Appl. Mater. Interfaces 11 (2019) 42780. https://doi.org/10.1021/acsami.9b14876

15 R. B. Mani and V. Anil: Mater. Lett. 232 (2018) 220. https://doi.org/10.1016/j.matlet.2018.08.114

16 A. Ghosh, P. Selvaraj, S. Sundaram, and T. K. Mallick: Sol. Energy 163 (2018) 537. https://doi.org/10.1016/ j.solener.2018.02.021 